\title{
Response of embryonic coral reef fishes (Pomacentridae: Amphiprion spp.) to noise
}

\author{
S. D. Simpson ${ }^{1, *}$, H. Y. Yan $^{2}$, M. L. Wittenrich ${ }^{3}$, M. G. Meekan ${ }^{4}$ \\ ${ }^{1}$ Institute of Evolutionary Biology, School of Biological Sciences, University of Edinburgh, Kings Buildings, \\ Edinburgh EH9 3JT, UK \\ ${ }^{2}$ Marine Research Station, Institute of Zoology, Academia Sinica, 23-10 Dahuen Road, Jiashi, I-Lan County 262, Taiwan, ROC \\ ${ }^{3}$ Fish Ecophysiology, Florida Institute of Technology, 150 W. University Avenue, Melbourne, Florida 32901, USA \\ ${ }^{4}$ Australian Institute of Marine Science, PO Box 40197, Casuarina, Darwin, Northern Territory 8010, Australia
}

\begin{abstract}
We investigated the ability of embryonic clownfishes Amphiprion ephippium and $A$. rubrocinctus to detect sound during incubation in benthic nests. The heart rates of embryos within eggs were monitored as the young fish were exposed to sounds in the range of 100 to $1200 \mathrm{~Hz}$ at levels of 80 to $150 \mathrm{~dB}$ (re $1 \mu \mathrm{Pa}$ at $1 \mathrm{~m}$ ) on each day of incubation. We found that, from $3 \mathrm{~d}$ after fertilisation, the heart rates of the embryos significantly increased when exposed to sound. As the embryos developed, a response in heart rate was found over a broader spectrum of sound (from 400 to $700 \mathrm{~Hz}$ at $3 \mathrm{~d}$ to a maximum of 100 to $1200 \mathrm{kHz}$ at $9 \mathrm{~d}$ after fertilisation) and sensitivity also increased, with response threshold minima at $700 \mathrm{~Hz}$ dropping from $139.1 \mathrm{~dB}$ at $3 \mathrm{~d}$ to $88.3 \mathrm{~dB}$ at $9 \mathrm{~d}$ after fertilisation. We discuss these findings with respect to recent work that demonstrates the importance of sound as a settlement cue in coral reef fishes.
\end{abstract}

KEY WORDS: Coral reef fishes $\cdot$ Embryos $\cdot$ Hearing $\cdot$ Sound $\cdot$ Heart rate $\cdot$ Amphiprion

\section{INTRODUCTION}

Of the approximately 100 families of teleost coral reef fishes, 36 are known to spawn their eggs in demersal nests on the reef, where they are brooded by adults (Shulman \& Bermingham 1995). While the duration of incubation and the competency of larvae at hatching vary considerably among species, in all reef fishes, the sensory systems of olfaction, hearing and vision are among the first organs to begin development in embryos after fertilisation (see review in Myrberg \& Fuiman 2002). This presumably occurs because these senses must attain some level of functionality if young larvae are to have any chance of avoiding predators and starvation upon hatching; however, the early development of these systems may also serve other functions. In some animals, the ability to sense environmental stimuli during incubation may imprint vital behavioural cues that are useful at older life-history stages. For example, imprinting of the chemical composition of sand on nesting beaches on embryonic sea turtles may determine the beach chosen by females for egg-laying in later life (Grassman 1993). In birds, the behaviour of young Muscovy ducklings Cairina moschata $f$. domestica has been shown to be influenced by maternal and other noises experienced prior to hatching (Hochel et al. 2002), while in coral reef fishes, Arvedlund et al. (1999) recently demonstrated that chemical cues may be imprinted on the brooded embryos of a clownfish Amphiprion melanopus prior to their hatching and export to the pelagic larval phase. This experience may ultimately influence the choice of settlement site when young fish return to reef environments.

It is not known whether other sensory systems such as vision and hearing might play a similar role for reef fishes, as no study has examined the functionality of these systems as embryos develop within eggs. However, we do know that sound may be an important navigational cue for reef fishes at the end of their larval 
life. Using a combination of light-traps, which are effective collectors of many settlement-stage reef fishes (Doherty 1987), and speakers broadcasting biological noise recorded from reefs (methods from Tolimieri et al. 2000), 2 recent studies have demonstrated the attraction of settlement-stage coral reef fishes to these sounds. Leis et al. (2003) found that some families of reef fishes (including pomacentrids) were attracted in greater numbers at some times to the traps with broadcast reef noises. In a similar study, Simpson et al. (2004) demonstrated a general attraction to reef sounds in presettlement larvae from 15 families of reef fishes. These included many that brood eggs in demersal nests. Other studies have shown orientation by late stage larvae to sound (Leis et al. 2002), implying that noise emanating from the reef may be used by larvae to guide their transition between pelagic and benthic environments.

The use of sound by larvae at the end of their pelagic phase and the rapid development of sensory systems in embryos raises the possibility that some late stage larvae respond to noises that were detected on the reef as brooded embryos. While intriguing, confirmation of this idea firstly requires demonstration that sensory organs attain a state of development sufficient for hearing to occur while young fish develop in the egg. Here, we use indirect methods to investigate whether there is a response to sound in the embryos of 2 coral reef anemone fishes, Amphiprion ephippium and $A$. rubrocinctus.

A major barrier to this type of study is that, to date, no standard technique exists for detecting sublethal responses to sound in embryos (i.e. sound levels that do not result in death). In our work, we use heart rate $(f \mathrm{H})$ to determine responses to sound by clownfish embryos. This trait has been employed as an indicator of health, stress and sensory ability in a wide range of embryonic, juvenile and adult fishes. For example, monitoring of $f \mathrm{H}$ has been used to determine the effect of temperature acclimation on rainbow trout Oncorhynchus mykiss (Aho \& Vornanen 2001) and triploid brown trout Salmo trutta (Altimiras et al. 2002, Mercier et al. 2002); the response to predator attacks in farmed and wild Atlantic salmon Salmo salar (Johnsson et al. 2001); to determine stress in rainbow trout from sound and light treatments (Kojima \& Soeda 1997); and to measure the threshold of hearing in juvenile red sea bream Pagrus major (Iwashita et al. 1999). Additionally, $f \mathrm{H}$ response has been used to examine sublethal effects of chemicals on fish embryos (Stouthart et al. 1996, Fitzsimons et al. 1999, Latif et al. 2001). By subjecting embryos to noises at each day of development and monitoring $f \mathrm{H}$, we aimed to determine when sounds could first be detected by embryos and to map the ontogenetic development of this response.

\section{MATERIALS AND METHODS}

We obtained spawns of 2 clownfishes from Oceans, Reefs and Aquariums (Harbor Branch Oceanographic Institute, Ft. Pierce, FL, USA). The clutches had been spawned on ceramic tiles, and were transported 2 (saddle anemonefish, Amphiprion ephippium) and 3 (red anemonefish, A. rubrocinctus) days post-fertilisation (d $\mathrm{PF}$ ). During transport, a fungicide, furozone green, was added to maintain the health of the eggs. Once installed, the tiles were agitated with a slow stream of bubbles in separate 371 aquariums, housed in a controlled temperature $\left(27^{\circ} \mathrm{C}\right)$ and light room $(13: 11 \mathrm{~h}$ light:dark cycle). Both clutches hatched on the night of 9 d PF (Fig. 1).

Experimental procedure. Each day, 5 eggs of each species were removed at random from each tile. The eggs were placed in separate Petri dishes in $2 \mathrm{~cm}$ of oxygenated water, and allowed to acclimatise at this pre-testing stage for $30 \mathrm{~min}$. To ensure that the embryos were healthy for use in the sound trials, the heart rate $(f \mathrm{H})$ of each was counted 5 times, over $15 \mathrm{~s}$ periods using a light microscope. Usually, $f \mathrm{H}$ was consistent among individual embryos at this time ( \pm 1 beat in $15 \mathrm{~s}$ ). Where a heart rate was abnormally high or low, the egg was replaced by another from the same clutch.

The following procedure was repeated on as many embryos each day as access to facilities and time allowed. A glass Petri dish $(6 \mathrm{~cm}$ in diameter, $2 \mathrm{~cm}$ in depth) containing an egg was placed on foam on an air table to remove building vibrations in a controlled acoustic environment (Industrial Acoustics). In this chamber, light and temperature were constant and external noise was eliminated. Ambient sound pressure inside the chamber was less than $60 \mathrm{~dB}$ (re $1 \mu \mathrm{Pa}$ at $1 \mathrm{~m})$. A compound microscope with a camera and video connection was used to view the embryo at a final magnification so that it diagonally filled the $34 \mathrm{~cm}$ screen of a monitor outside the chamber (Fig. 2). Using this set up, the beating heart of the embryo was clearly visible through the transparent egg case. Heartbeats were counted from the TV monitor and recorded using a Panasonic AG-1970 sVHS video player.

After 10 min of acclimatisation in the testing arena, the base $f \mathrm{H}$ of the embryo (per $15 \mathrm{~s}$ ) was counted at least 5 times by 2 observers (S.D.S. and M.L.W.). Counts of observers were compared prior to experimental treatments to ensure consistency in the counts. The embryo was then treated with a sound stimulus generated using a Tucker-Davis Technologies (TDT) modular rack system controlled by an optically-linked Pentium III, $350 \mathrm{MHz}$ desktop computer consisting of a TDT board, and running TDT BioSig ${ }^{\mathrm{TM}}$ software. The sound treatments were emitted from a speaker (Pioneer, frequency response $19 \mathrm{~Hz}$ to $15 \mathrm{kHz}$ ) $1 \mathrm{~m}$ above 
the egg for $75 \mathrm{~s}$ as $20 \mathrm{~ms}$ tone bursts of a specified frequency. During the treatment, five $f \mathrm{H}$ readings over $15 \mathrm{~s}$ periods were taken. Approximately $1 \mathrm{~min}$ following the treatment, at least 5 new base rates were recorded over a period in excess of $3 \mathrm{~min}$.

The test frequencies we used were 100, 400, 700, 1000 and $1200 \mathrm{~Hz}$ pure tone sound. On the first day, the initial Sound Pressure Levels (SPL) used were the maximums that the equipment could generate. Since the sound was emitted in air, but received by the embryos underwater, we used a hydrophone (Celesco LC-10) in a similar depth of water to calibrate our treatments to the resultant sound pressure levels (re $1 \mu \mathrm{Pa}$ at $1 \mathrm{~m}$ ) at each frequency received by the embryos;
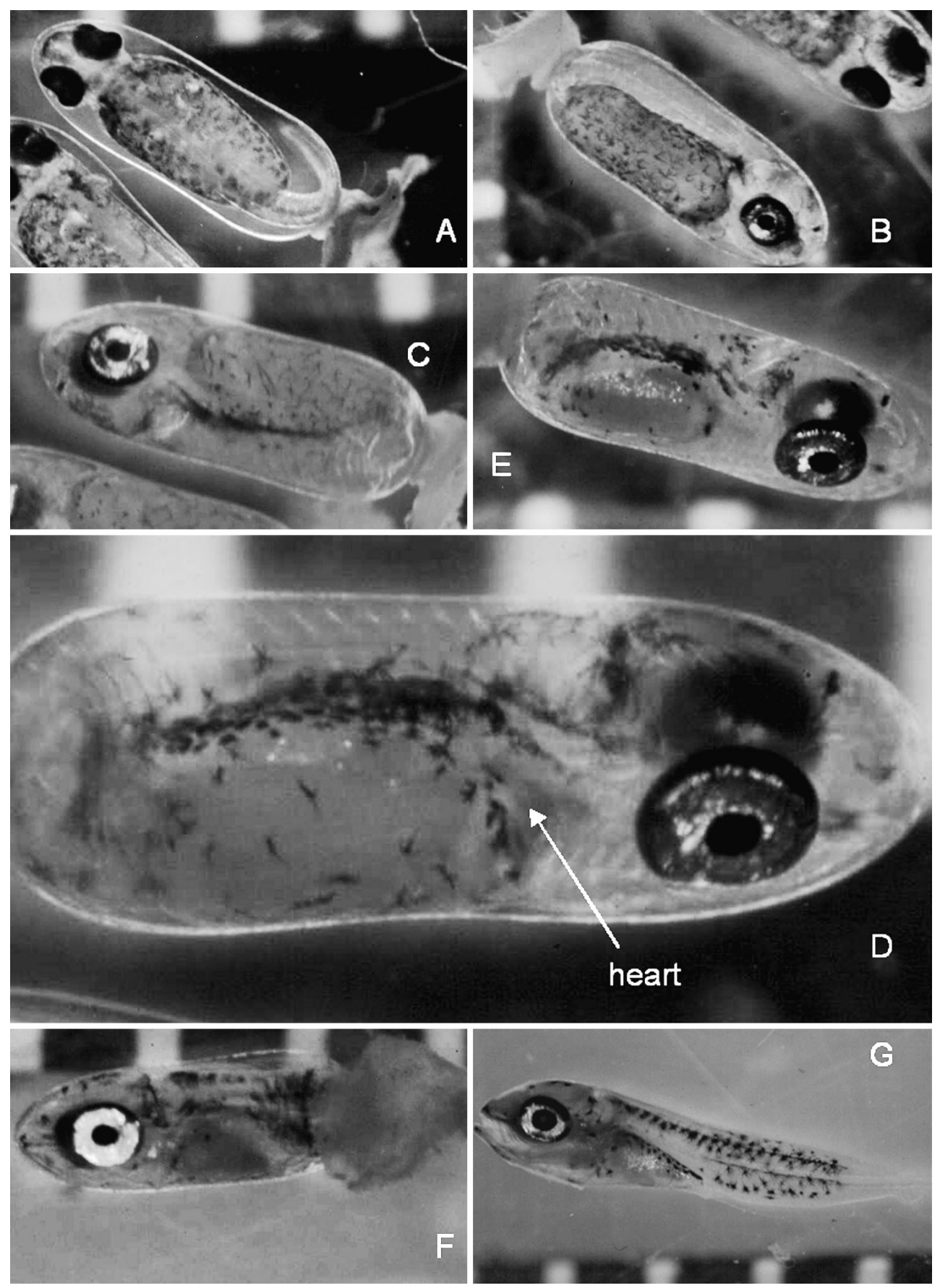

Fig. 1. Amphiprion ephippium. Embryonic development (A) 4 d post-fertilisation (d PF), (B) 5 d PF, (C) 6 d PF, (D) 7 d PF (enlarged to show heart region), (E) $8 \mathrm{~d} \mathrm{PF},(\mathrm{F}) 9 \mathrm{~d} \mathrm{PF},(\mathrm{G}) 12 \mathrm{~h}$ post-hatching. Each division of scale bars behind the subjects is $1 \mathrm{~mm}$ 


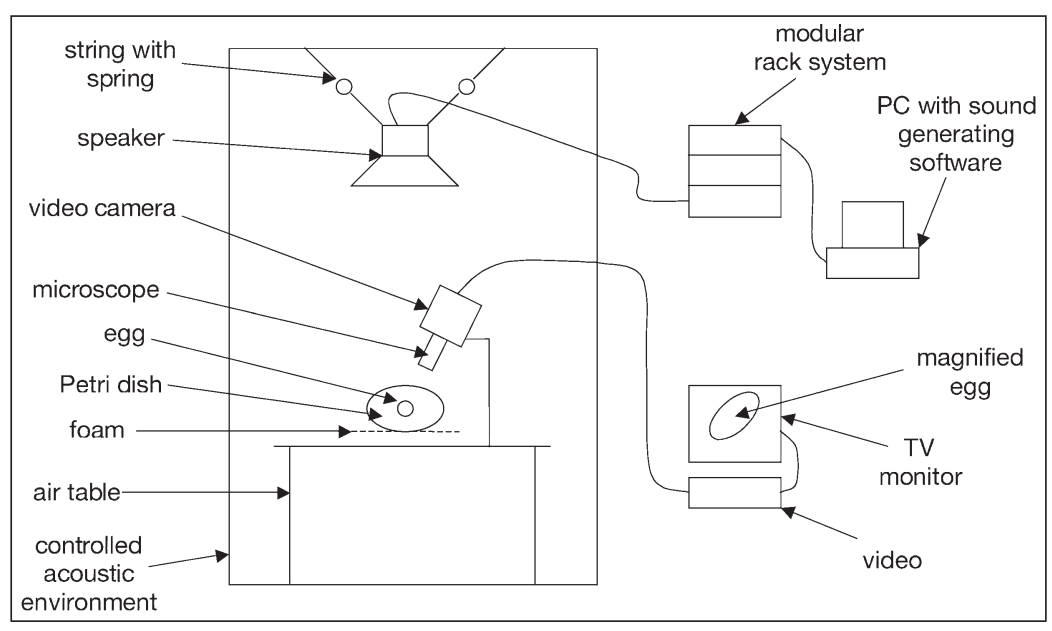

Fig. 2. Experimental set-up showing an egg in the treatment chamber outside the $95 \%$ confidence intervals (mean $\pm 2 \mathrm{SD}$ ) of the previous mean base $f \mathrm{H}$ reading. In our study, we compared the mean treatment $f \mathrm{H}$ with both the pre- and post-treatment mean base $f \mathrm{H}$, and accepted a response in heart rate only if both were significantly different from the treatment mean. In a second approach, we applied the Mann-Whitney $U$-test to the treatment $f \mathrm{H}$ readings versus the pre- and post-treatment $f \mathrm{H}$ readings (Sokal \& Rohlf 1995). This test detected potential responses less frequently, presumably because our sample sizes $(n=5)$ were low and the variation among replicate trials was very low. Ultimately, we accepted that there had been a response in heart these SPLs were in the range of 132 to $150 \mathrm{~dB}$ (re $1 \mu \mathrm{Pa}$ at $1 \mathrm{~m}$ ), depending on frequency. These frequencies and SPLs are comparable to natural noises on the reefs, which are predominantly in the range of $20 \mathrm{~Hz}$ to $15 \mathrm{kHz}$, and can exceed $156 \mathrm{~dB}$ (re $1 \mu \mathrm{Pa}$ at $1 \mathrm{~m}$ ) at the source (Myrberg et al. 1986, McCauley \& Cato 2000).

On subsequent days, we initially tested each frequency at $10 \mathrm{~dB}$ less than the threshold on the previous day if there had been a statistically significant response; otherwise we remained at the maximum output. The $f \mathrm{H}$ readings were entered into a computer spreadsheet, such that we could visually determine elevated $f \mathrm{H}$ responses when they occurred. This crude analysis was later tested statistically (see below). Where a response appeared to have occurred, we then allowed the embryo to return to a rest $f \mathrm{H}$, before then attenuating the SPL at the same frequency by $10 \mathrm{~dB}$ in the subsequent treatment. This process was repeated until no response was seen. We then increased the SPL to the previous level, to confirm that the response was visible.

Observer bias. To test for possible observer bias, 2 naïve readers were shown sections of video recordings of heart beats from 4 embryos. These were chosen to include base counts, periods of treatment where significant responses of heart beat rates were seen, and periods of treatment where there were no significant responses. Naïve readers were then asked to count heartbeats on these tapes. By playing back the videos without the sound track, readers were not aware when sound treatments and controls were in progress.

Statistical analyses. To identify responses in $f \mathrm{H}$ to sound treatments, we analysed the data in 2 ways. The first method, used in previous studies of heart rates and hearing (e.g. Popper et al. 1973), set a response criteria that the mean of the treatment $f \mathrm{H}$ readings should lie rate to sound only where there was a significant ( $p<$ 0.05 ) rise in $f \mathrm{H}$ during the treatment and a fall in $f \mathrm{H}$ after the treatment, using both statistical analyses.

In order to test for observer bias, counts of heart rates obtained from naïve and experienced observers were compared using regression analyses and Wilcoxon matched-pairs tests (Sokal \& Rohlf 1995).

\section{RESULTS}

It was possible to identify a $f \mathrm{H}$ response to sound in the embryos of both species from 3 to $5 \mathrm{~d}$ postfertilisation ( $\mathrm{d} \mathrm{PF}$ ). A change in $f \mathrm{H}$ that was statistically significant was in the region of $+10 \%$; so for Amphiprion rubrocinctus, the heart rate increased from 180 to 200 beats per minute at $5 \mathrm{~d}$ PF during a significant treatment. In total, $21 \mathrm{~A}$. ephippium embryos were tested in the period 3 to $9 \mathrm{~d}$ PF (day:number; 3:3; $4: 2 ; 5: 4 ; 6: 3 ; 7: 3 ; 8: 4 ; 9: 2)$, and 14 A. rubrocinctus embryos were tested in the period 5 to $9 \mathrm{~d}$ PF (day:number; $5: 2 ; 6: 3 ; 7: 3 ; 8: 2 ; 9: 4)$. At least 2 embryos were examined from each species each day. On 4 occasions, at the upper limits of the output of the equipment, responses were not seen in all individuals, but always in more than half (A. ephippium: 2 of 3 at $400 \mathrm{~Hz}, 3 \mathrm{~d} \mathrm{PH}$; 3 of 4 at $1 \mathrm{kHz}, 5 \mathrm{~d} \mathrm{PF}$; 2 of 3 at $1200 \mathrm{~Hz}, 6 \mathrm{~d} \mathrm{PF}$; and $A$. rubrocinctus: 2 of 3 at $100 \mathrm{~Hz}, 6 \mathrm{~d} \mathrm{PF}$ ). These events are indicated in the respective figures.

As larvae developed, $f \mathrm{H}$ became increasingly sensitive to sound treatments and the spectral range at which a response was recorded also broadened (Fig. 3). A $f \mathrm{H}$ response to mid-range sounds (400 and $700 \mathrm{~Hz}$ ) was seen in Amphiprion ephippium from $3 \mathrm{~d}$ PF onwards (Fig. 3A). At 400 and $700 \mathrm{~Hz}$, the sensitivity of $f \mathrm{H}$ increased (thresholds dropped) steadily 

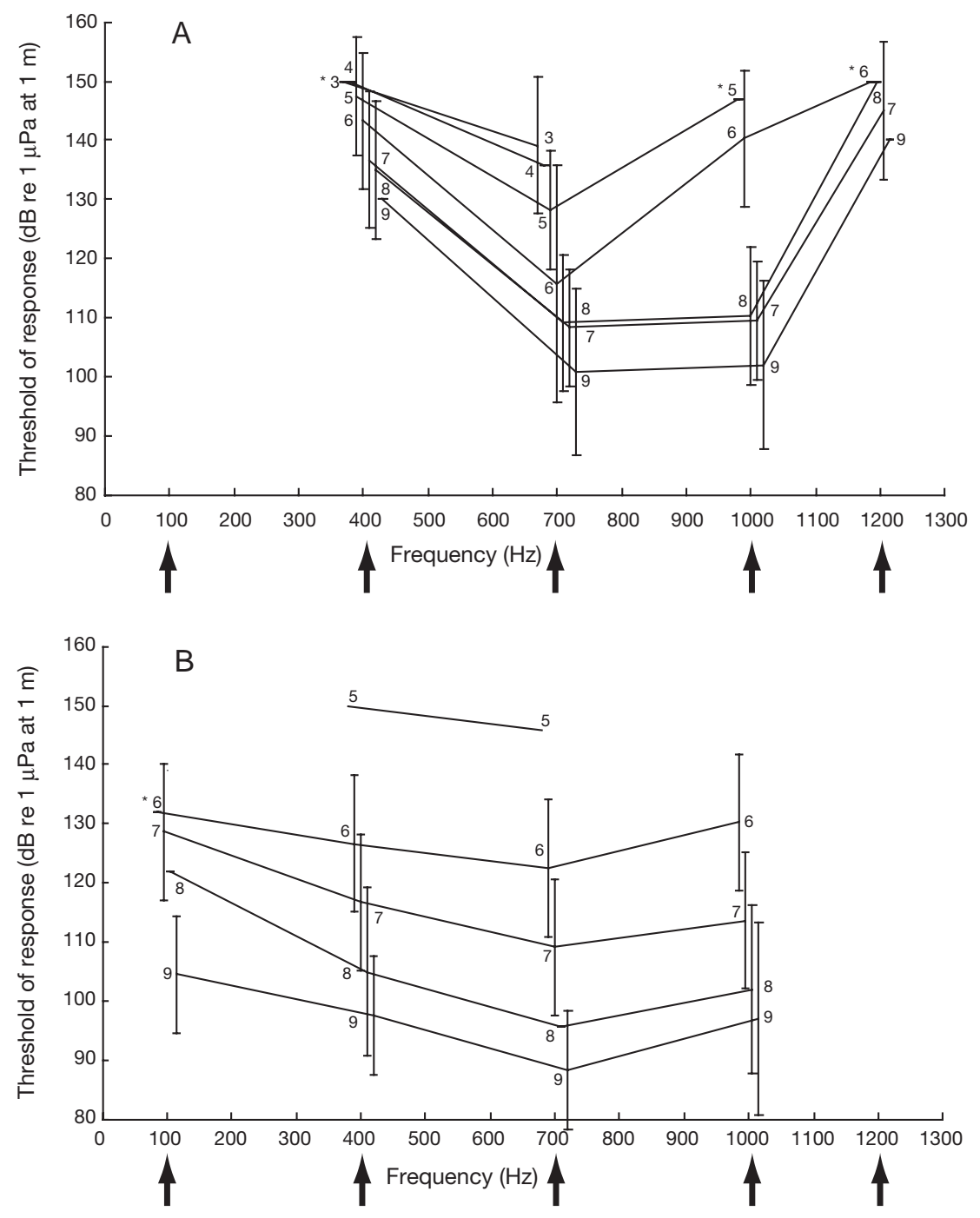

Fig. 3. (A) Amphiprion ephippium and (B) A. rubrocinctus. Development of the threshold of response to sound treatments by embryos during development. Arrows indicate the test frequencies; data labels indicate the age of the embryo (days postfertilisation) and error bars show 95\% confidence limits. Notice no significant response at $100 \mathrm{~Hz}$ for $A$. ephippium, while no significant response at $1200 \mathrm{~Hz}$ for A. rubrocinctus. Asterisks indicate incomplete data sets (see note in text)
Almost identical $f \mathrm{H}$ values were recorded by the 2 naïve readers, and the correlations between our readings and those of both the naïve readers were very strong and almost equivalent (Fig. 4). Using the Wilcoxon matched-pairs test, we found no significant differences between our readings and those of the naïve readers ( $\mathrm{n}=21, \mathrm{p}>0.05)$. This result confirms that our readings of heart rates do not have any preconceived bias.

\section{DISCUSSION}

Our study provides the first evidence that embryonic fish are capable of responding to sound, and that the sensitivity and spectral range of response increase in the period leading up to hatching. It is important to recognise that our measurements of heart rate response only provide an indirect estimate of acoustic sensitivity of young fish, in contrast to techniques such as Auditory Brainstem Response (e.g. Scholik \& Yan 2001) that more directly measure hearing by placing electrodes to detect nervous responses near the brainstem of the fish during sound treatments. However, since other stimuli were controlled, the changes in our measures of $f \mathrm{H}$ were certainly a response to acoustic stimuli. It was beyond the scope of this study to infer the exact neurological mechanism by which fish sense sounds at different times throughout their development. Matching the development of a response to sound throughout embryonic development. A response at $1 \mathrm{kHz}$ became significant $5 \mathrm{~d} \mathrm{PF}$, and the threshold dropped rapidly thereafter, while at $1.2 \mathrm{kHz}$, a response was first seen $6 \mathrm{~d} P F$, although the threshold dropped relatively little throughout the rest of the period of embryonic development. No $f \mathrm{H}$ response was ever recorded at $100 \mathrm{~Hz}$.

A similar pattern of increasing sensitivity and broadening spectral range of $f \mathrm{H}$ response was observed throughout the embryonic development of Amphiprion rubrocinctus (Fig. 3B). In contrast to A. ephippium, however, responses were observed for $100 \mathrm{~Hz}$ treatments from $6 \mathrm{~d}$ PF onwards, but were never observed for $1.2 \mathrm{kHz}$ treatments. with morphological changes in the sensory system using histology would be a very interesting area of further study.

There is the possibility that the use of bubbles to aerate the eggs in the aquariums may potentially affect the hearing of the embryos prior to testing. Although bubbling was kept to a minimum, without jeopardising the health of the eggs, there was inevitably some resultant ambient noise in the aquariums. Since this noise could damage the hearing of the fish, this would only bias our data towards not finding a response to sound, thus increasing the estimates of the threshold of hearing we observed. Due to this factor, and the use of conservative statistical methods, our findings should be 


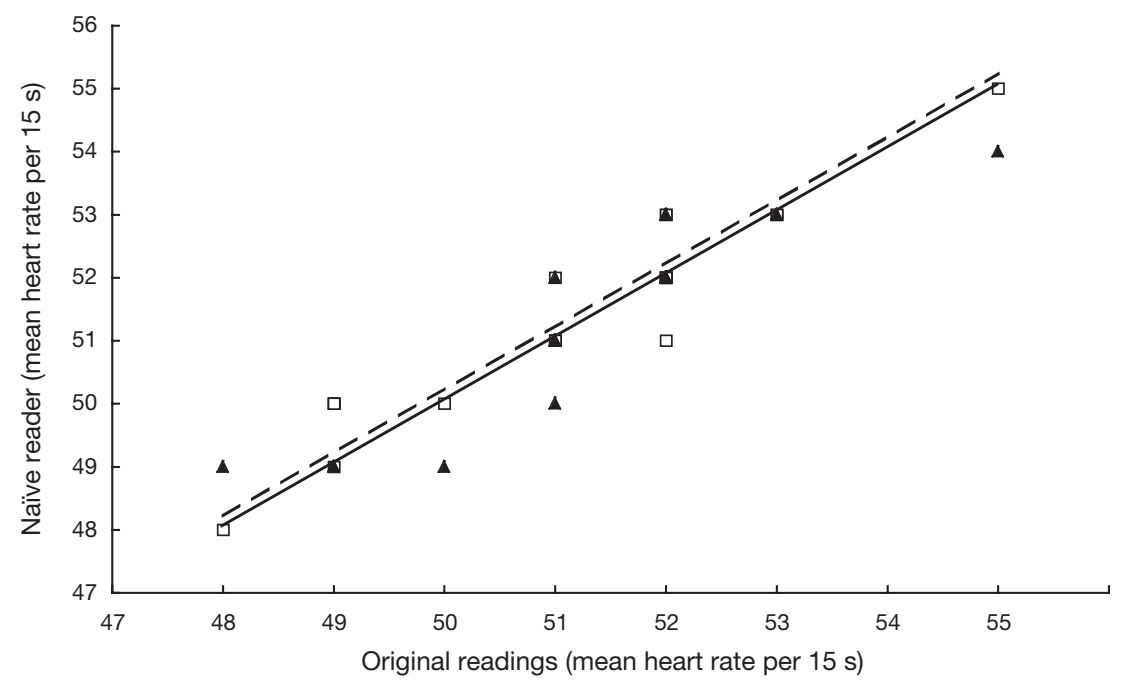

Fig. 4. Regression analysis of the heart-rate readings versus those of 2 naïve readers $(\mathrm{n}=21)$. Readings by S.D.S./M.L.W. versus those of Reader 1: $\boldsymbol{\Delta}-\boldsymbol{\Lambda}, y=$ $1.0009 x, R^{2}=0.88$; Readings by S.D.S./M.L.W. versus those of Reader 2: $\square---\square$, $y=1.0045 x, R^{2}=0.89$

taken as underestimates of the real thresholds of hearing of embryos in nature.

This study shows that the embryos of the $2 \mathrm{Am}$ phiprion spp. responded to different spectral ranges of sound. Heart rates of $A$. ephippium embryos increased when they were subjected to sounds of $1.2 \mathrm{kHz}$ from $6 \mathrm{~d}$ $\mathrm{PF}$ onwards, while a response to such frequencies was never seen in $A$. rubrocinctus. Conversely, $A$. ephippium never responded to treatments at $100 \mathrm{~Hz}$, whereas $A$. rubrocinctus responded at $100 \mathrm{~Hz}$ after $6 \mathrm{~d}$ PF. It is possible that this result is linked to the ecology of these species. $A$. ephippium is found naturally on sheltered reefs, often in silty, low visibility coastal waters, especially in enclosed bays (Allen 1991). There are few sources of low frequency noise in these environments, whereas the relatively high energy coastal reefs inhabited by $A$. rubrocinctus have a constant source of low frequency noise due to breaking waves (Deane \& Stokes 2002).

Most biologically-produced sound in the marine environment is in the range $20 \mathrm{~Hz}$ to $15 \mathrm{kHz}$ (although snapping shrimp crackle and cetacean sonar is produced at frequencies exceeding $200 \mathrm{kHz}$ ), and fish calls generally fall in the range of $50 \mathrm{~Hz}$ to $3 \mathrm{kHz}$ (McCauley 2001). For example, the territorial Western Atlantic pomacentrid Stegastes partitus is known to produce sounds in the range of $350 \mathrm{~Hz}$ to $1 \mathrm{kHz}$ during reproductive activity (Myrberg et al. 1986), while in Australia McCauley \& Cato (2000) recorded nocturnal planktivores (probably representatives of Priacanthidae and Holocentridae) producing popping noises in the range of 400 to $700 \mathrm{~Hz}$, an unknown fish 'banging' at 144 to $147 \mathrm{~Hz}$, and the teraponid Terapon theraps 'trumpeting' at 110 to $140 \mathrm{~Hz}$.

Response frequencies and thresholds of the embryos overlap the acoustic signatures produced by these fish. By overlaying frequency ranges and the source pressure level of the noises described above on response plots of embryos (Fig. 5), it becomes clear that embryos within nests will experience a suite of biological noise during their development (Fig. 5). The reproductive noises of pomacentrids (assuming Pomacentrus partitus is typical of pomacentrids) would be detected by Amphiprion ephippium embryos during the last $3 \mathrm{~d}$ of embryonic development (A in Fig. 5), while the upper end of the Terapon theraps trumpeting would be detected during the last $4 \mathrm{~d}$ of incubation (B in Fig. 5). Moreover, the popping of the priacanthids and the holocentrids (C and D in Fig. 5)

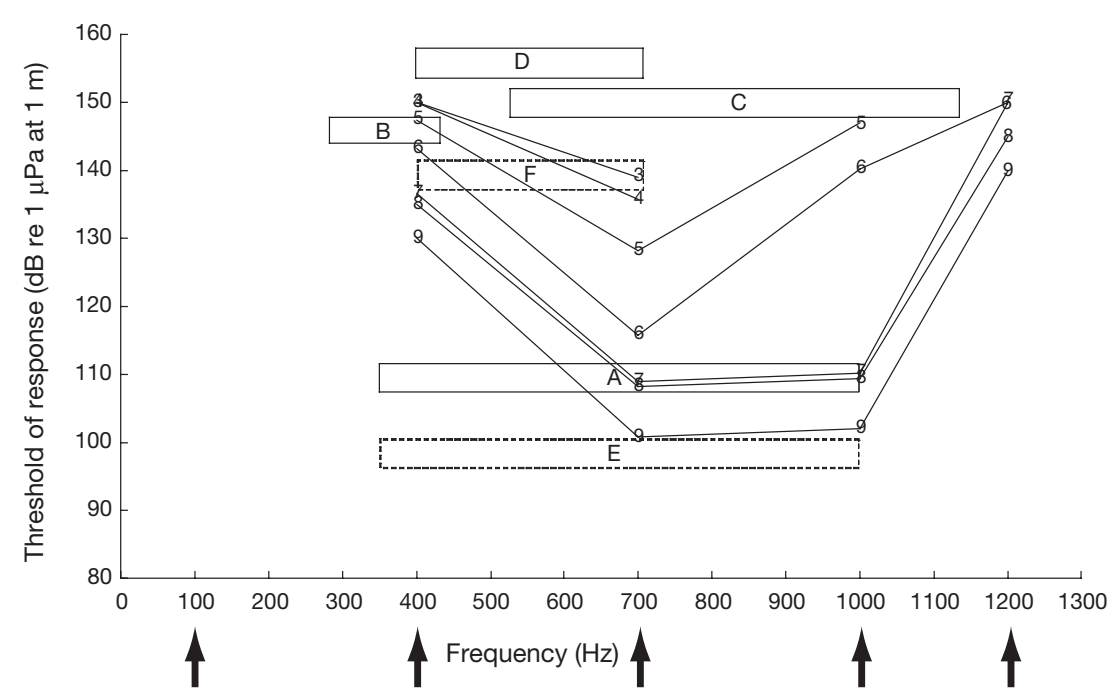

Fig. 5. Amphiprion ephippium. Development of the threshold of response to sound treatments by embryos with sources of biological noise 'mapped' on the same axes according to the published source pressure levels (re $1 \mu \mathrm{Pa}$ at $1 \mathrm{~m}$ ) and frequencies. A: Pomacentrus partitus (in Myrberg et al. 1986); B: Terapon theraps; C: 'banging' (source unknown); D: nocturnal planktivores (B-D in McCauley \& Cato 2000); E: P. partitus at $10 \mathrm{~m}$ from source; F: nocturnal planktivores at $100 \mathrm{~m}$ from source (E \& F calculated using A \& D) 
would be experienced throughout development. These fishes (excluding P. partitus but including most of the genus Pomacentrus) are widespread and abundant on Indo-Pacific coral reefs where anemone fishes occur.

While the overlap between response thresholds and biological sound on coral reefs is intriguing, it is not the only factor that must be considered in determining whether developing embryos can detect reef sounds. As sound pressure levels may attenuate rapidly on the reef, the distance of the nest from sound sources will influence hearing ability. For example, if a pomacentrid produced noise more than $10 \mathrm{~m}$ from a nest, not even the $9 \mathrm{~d}$ PF embryos would detect it (E in Fig. 5). However, the popping noise of priacanthids and holocentrids would still be within the thresholds of a $7 \mathrm{~d}$ PF embryo, if it were produced at a distance of $100 \mathrm{~m}$ from eggs ( $\mathrm{F}$ in Fig. 5). This suggests that the noise experienced by embryos is very dependent on the location of the nest. Further complicating the issue is the fact that, unlike our study where the eggs were kept in a controlled sound-proof environment, the reef is generally a noisy place. Snapping shrimp, feeding parrotfishes (Scaridae), breaking waves and rain combine to produce background noise, which also varies with location and over time, although probably not with the same distinctive qualities as fish calling. The extent to which embryos are capable of isolating single sound sources from the background noise (the 'Cocktail Party Problem', Cherry 1953) remains to be determined, and the critical thresholds needed to do this are largely mysterious in higher mammals, let alone in embryonic fish. From the perspective of settlement-stage fishes navigating to reefs, it is likely that this background noise will be detectable from further away, and may provide the first general acoustic cues before more distinctive location-specific sounds can be detected. Further studies to investigate the acoustic heterogeneity amongst and within reefs, and how this changes over time, will greatly benefit our understanding of the availability of sound cues, and the specificity that the quality of this information can provide.

The incubation period of eggs of anemone fishes is long in comparison to most other species within the family ( $9 \mathrm{~d}$ versus 4 to $5 \mathrm{~d}$ for most other species in the genus Pomacentrus, Allen 1991), so that anemone fish larvae at hatching are relatively well developed. This may mean that the ability of embryos to detect sound is not necessarily shared among all members of the family, nor that it is necessarily typical of other reef fishes that brood eggs. Furthermore, we do not know at present what function (if any) this ability may perform. It is possible that hearing may aid survivorship of plankton after hatching in some manner. However, hearing also fulfils an important prerequisite if these fish are to have the potential to imprint on sound cues that guide their later settlement. Exploration of this possibility will require a detailed description of the 'soundscape' of coral reefs and experimental studies that attempt to imprint developing embryos, and then follow their subsequent settlement patterns. These topics are subjects of future work.

Acknowledgements. We thank V. Rado at ORA for providing the Amphiprion spawns, A. Scholik and A. Wigginton for being naïve readers, R. McCauley for use of his data, and C. Dytham for statistical advice. The animal-use protocol for this study was approved by the University of Kentucky Institutional Animal Care and Use Committee (00217L2001). This work was supported by a Natural Environment Research Council postgraduate fellowship and the British Association (to S.D.S.), the Institute of Museum and Library Service of the US Department of Education, the National Institute of Mental Health, the National Organization for Hearing Research, the Kentucky Water Resources Research Institute, and a University of Kentucky Research Committee grant (to H.Y.Y.), and the Australian Institute of Marine Science grant (to M.G.M).

\section{LITERATURE CITED}

Aho E, Vornanen M (2001) Cold acclimation increases basal heart rate but decreases its thermal tolerance in rainbow trout (Oncorhynchus mykiss). J Comp Physiol B 171: 173-179

Allen GR (1991) Damselfishes of the world. Mergus Publishers, Melle

Altimiras J, Axelsson M, Claireaux G, Lefrancois C, Mercier C, Farrell AP (2002) Cardiorespiratory status of triploid brown trout during swimming at two acclimation temperatures. J Fish Biol 60:102-116

Arvedlund M, McCormick MI, Fautin DG, Bildsoe M (1999) Host recognition and possible imprinting in the anemonefish Amphiprion melanopus (Pisces: Pomacentridae). Mar Ecol Prog Ser 188:207-218

Cherry EC (1953) Some experiments on the recognition of speech, with one and two ears. J Acoust Soc Am 25: 975-979

Deane GB, Stokes MD (2002) Scale dependence of bubble creation mechanisms in breaking waves. Nature 418: 839-844

Doherty PJ (1987) Light-traps: selective but useful devices for quantifying the distributions and abundances of larval fishes. Bull Mar Sci 41:423-431

Fitzsimons JD, Brown SB, Honeyfield DC, Hnath JG (1999) A review of early mortality syndrome (EMS) in great lakes salmonids: relationship with thiamine deficiency. Ambio 28:9-15

Grassman M (1993) Chemosensory orientation behavior in juvenile sea-turtles. Brain Behav Evol 41:224-228

Hochel J, Pirow R, Nichelmann M (2002) Development of heart rate responses to acoustic stimuli in Muscovy duck embryos. Comp Biochem Physiol A 131:805-816

Iwashita A, Sakamoto M, Kojima T, Watanabe Y, Soeda H (1999) Growth effects on the auditory threshold of red sea bream. Nippon Suisan Gakkaishi 65:833-838

Johnsson JI, Hojesjo J, Fleming IA (2001) Behavioural and heart rate responses to predation risk in wild and domesticated Atlantic salmon. Can J Fish Aquat Sci 58:788-794

Kojima T, Soeda H (1997) Heart rate change of rainbow trout under long period illumination and sound stimuli. Nippon Suisan Gakkaishi 63:905-911 
Latif MA, Bodaly RA, Johnston TA, Fudge RJP (2001) Effects of environmental and maternally derived methylmercury on the embryonic and larval stages of walleye (Stizostedion vitreum). Environ Pollut 111:139-148

Leis JM, Carson-Ewart BM, Cato DH (2002) Sound detection in situ by the larvae of a coral-reef damselfish (Pomacentridae). Mar Ecol Prog Ser 232:259-268

Leis JM, Carson-Ewart BM, Hay AC, Cato DH (2003) Coralreef sounds enable nocturnal navigation by some reef-fish larvae in some places at some times. J Fish Biol 63:724-737

McCauley RD (2001) Biological sea noise in northern Australia: patterns of fish calling. $\mathrm{PhD}$ thesis, James Cook University, Townsville

McCauley RD, Cato DH (2000) Patterns of fish calling in a nearshore environment in the Great Barrier Reef. Phil Trans R Soc Lond B 355:1289-1293

Mercier C, Axelsson M, Imbert N, Claireaux G, Lefrancois C, Altimiras J, Farrell AP (2002) In vitro cardiac performance in triploid brown trout at two acclimation temperatures. J Fish Biol 60:117-133

Myrberg AA, Fuiman LA (2002) The sensory world of coral reef fishes. In: Sale PF (ed) Coral reef fishes: dynamics and diversity in a complex ecosystem. Elsevier Science, New York, p 123-148

Editorial responsibility: Otto Kinne (Editor-in-Chief), Oldendorf/Luhe, Germany
Myrberg AA, Mohler M, Catala JD (1986) Sound production by males of a coral reef fish (Pomacentrus partitus): its significance to females. Anim Behav 34:913-923

Popper AN, Chan ATH, Clarke NL (1973) An evaluation of methods for behavioral investigations of teleost audition. Behav Res Meth Instr 5:470-472

Scholik AR, Yan HY (2001) Effects of underwater noise on auditory sensitivity of a cyprinid fish. Hear Res 152:17-24

Shulman MJ, Bermingham E (1995) Early life histories, ocean currents, and the population genetics of Caribbean reef fishes. Evolution 49:897-910

Simpson SD, Meekan MG, McCauley RD, Jeffs A (2004) Attraction of settlement-stage coral reef fishes to reef noise. Mar Ecol Prog Ser 276:263-268

Sokal RR, Rohlf FJ (1995) Biometry, 3rd edn. WH Freeman, New York

Stouthart X, Haans JLM, Lock RAC, Bonga SEW (1996) Effects of water $\mathrm{pH}$ on copper toxicity to early life stages of the common carp (Cyprinus carpio). Environ Toxicol Chem 15:376-383

Tolimieri N, Jeffs A, Montgomery JC (2000) Ambient sound as a cue for navigation by the pelagic larvae of reef fishes. Mar Ecol Prog Ser 207:219-224

Submitted: January 29, 2004; Accepted: November 16, 2004 Proofs received from author(s): January 25, 2005 